\title{
AN ADOMIAN DECOMPOSITION METHOD FOR SOLVING LIÉNARD EQUATIONS IN GENERAL FORM
}

\author{
M. NILI AHMADABADI ${ }^{\bowtie}$ and F. M. MAALEK GHAINI ${ }^{1}$
}

(Received 21 October, 2007; revised 31 August, 2009)

\begin{abstract}
In this study, Liénard equations in their general form are treated using the Adomian decomposition method. The special structure of the Liénard equation is exploited to obtain a numerically efficient algorithm suitable for solution by a computer program.

2000 Mathematics subject classification: primary 65L05; secondary 41A10.

Keywords and phrases: Liénard equation, numerical solutions, Adomian decomposition method, Adomian polynomials.
\end{abstract}

\section{Introduction}

Let $f$ and $g$ be two continuously differentiable functions on $\mathbb{R}$, with $f$ an even function and $g$ an odd function. A second-order ordinary differential equation of the form

$$
y^{\prime \prime}+f(y) y^{\prime}+g(y)=0
$$

is called a Liénard equation. During the development of radio and vacuum tubes, Liénard equations were intensively studied as they can be used to model oscillating circuits. The Liénard equation is not only regarded as a generalization of the damped pendulum equation or a damped spring-mass system (where $f(y) y^{\prime}$ is the damping force and $g(y)$ is the restoring force), but is also used in nonlinear models of many physically significant fields for different choices of $f(y)$ and $g(y)$. Under certain additional assumptions, Liénard's theorem guarantees the existence of a limit cycle for such a system [21].

The equation governing the van der Pol oscillator [13],

$$
y^{\prime \prime}-\mu\left(1-y^{2}\right) y^{\prime}+y=0,
$$

can be regarded as a special case of $(1.1)$ in which $f(y)=-\mu\left(1-y^{2}\right)$ and $g(y)=y$. Here we emphasize the presence of the $y^{\prime}$ term in (1.2).

\footnotetext{
${ }^{1}$ Department of Mathematics, Yazd University, Yazd 89195-741, Iran; e-mail: mnili@yazduni.ac.ir, maalek@yazduni.ac.ir.

(C) Australian Mathematical Society 2010, Serial-fee code 1446-1811/2010 \$16.00
} 
Recently, in [16] the Adomian decomposition method (ADM) [5, 6, 18] was implemented to solve Liénard equations of the type

$$
y^{\prime \prime}+l y+m y^{3}+n y^{5}=0,
$$

where $l, m$ and $n$ are real coefficients.

In this paper we use the ADM in an efficient way to solve Liénard equations (1.1) in which $f(x)$ and $g(x)$ are even and odd functions, respectively. When $f(y)$ is not constant, finding a closed form solution for the Liénard equation-when one exists-is very difficult [3]. This indicates the need for an efficient iterative method.

\section{The Adomian decomposition method}

The ADM is a powerful device for solving functional equations. In this method, special kinds of polynomials are used which are called Adomian polynomials. The solution is given by an infinite series in which each term is easily obtained. Cherruault and his collaborators have proved the convergence of the method when applied to certain functional equations $[1,9]$. Consider the functional equation

$$
y=N(y)+f
$$

where $f$ is a known function, $N$ is a nonlinear operator and $y$ is an unknown function. In order to use the Adomian decomposition method we define

$$
y=\sum_{n=0}^{\infty} y_{n}
$$

and the nonlinear function is decomposed as

$$
N(y)=\sum_{n=0}^{\infty} A_{n},
$$

where the $A_{n}$ are Adomian polynomials. These depend only on $y_{0}, y_{1}, \ldots, y_{n}$ and are given by [3]

$$
A_{n}=\frac{1}{n !} \frac{d^{n}}{d \lambda^{n}}\left[N\left(\sum_{i=0}^{\infty} \lambda^{i} y_{i}\right)\right]_{\lambda=0}, \quad n=0,1,2, \ldots
$$

The first few Adomian polynomials are given by

$$
A_{0}=N\left(y_{0}\right), \quad A_{1}=y_{1} N^{\prime}\left(y_{0}\right), \quad A_{2}=y_{2} N^{\prime}\left(y_{0}\right)+\frac{1}{2} y_{1}^{2} N^{\prime \prime}\left(y_{0}\right) .
$$

Substituting (2.2) and (2.3) into (2.1) yields

$$
\sum_{n=0}^{\infty} y_{n}=\sum_{n=0}^{\infty} A_{n}+f
$$

The Adomian technique utilizes the recursions

$$
y_{0}=f, \quad y_{n+1}=A_{n}, \quad n=0,1,2, \ldots
$$


and is equivalent to determining the sequence

$$
s_{n}=y_{1}+y_{2}+\cdots+y_{n},
$$

by using the iterative scheme

$$
\left\{\begin{array}{l}
s_{0}=0 \\
s_{n+1}=N\left(y_{0}+s_{n}\right)
\end{array}\right.
$$

associated with the functional equation

$$
s=N\left(y_{0}+s\right),
$$

which can be solved by the Adomian scheme [11]

$$
\left\{\begin{array}{l}
s_{0}=0 \\
s_{n+1}=A_{n}\left(y_{0}, y_{1}, \ldots, y_{n}\right)
\end{array}\right.
$$

The following theorem provides a sufficient condition for the convergence of the ADM.

TheOREM 2.1 ([15]). Let $N$ be an operator from a Hilbert space $H$ into $H$ and $y$ be the exact solution of (2.1). Then $\sum_{n=0}^{\infty} y_{n}$ converges to $y$ when there exists an $0 \leq \alpha<1$ such that $\left\|y_{i+1}\right\| \leq \alpha\left\|y_{i}\right\|$ for $i=0,1,2, \ldots$.

For the order of convergence we have the following theorem.

TheOREM 2.2 ([7]). Suppose that $N \in C^{p}[a, b]$. If $N^{(k)}\left(y_{0}+s\right)=0, k=$ $0,1, \ldots, p-1$ and $N^{(p)}\left(y_{0}+s\right) \neq 0$, then the convergence of the sequence $s_{n}$ is of order $p$.

\section{Description of the method}

In this section we present an ADM for solving the initial value problem

$$
y^{\prime \prime}+f(y) y^{\prime}+g(y)=0, \quad y(0)=a, \quad y^{\prime}(0)=b,
$$

where $f$ and $g$ are even and odd continuous functions, respectively. Solution of this equation first requires some simplifying manipulation. Integrating both sides from 0 to $x$ and using the initial conditions gives

$$
y^{\prime}(x)=b-\int_{0}^{x} f(y(s)) y^{\prime}(s) d s-\int_{0}^{x} g(y(s)) d s .
$$

On the other hand,

$$
\int_{0}^{x} f(y(s)) y^{\prime}(s) d s=\int_{a}^{y} f(t) d t=F(y)-F(a),
$$

in which $F(t)$ represents an antiderivative of $f(t)$. Substituting (3.3) into (3.2) yields

$$
y^{\prime}(x)=(b+F(a))-F(y)-\int_{0}^{x} g(y(s)) d s .
$$


Again, we integrate both sides of (3.4) from 0 to $x$ to obtain

$$
y(x)=a+(b+F(a)) x-\int_{0}^{x} F(y(s)) d s-\int_{0}^{x} \int_{0}^{t} g(y(s)) d s d t .
$$

Now we utilize the ADM on the nonlinear integral equation (3.5). Let

$$
y(x)=\sum_{n=0}^{\infty} y_{n}(x) \text {. }
$$

Therefore, we can write

$$
F(y)=\sum_{n=0}^{\infty} A_{n} \quad \text { and } \quad g(y)=\sum_{n=0}^{\infty} B_{n},
$$

where $A_{n}$ and $B_{n}$ are Adomian polynomials depending on $F$ and $g$, respectively [6]. Several authors have proposed efficient methods for calculating Adomian polynomials $[8,19,20]$.

Substituting (3.6) and (3.7) into (3.5),

$$
\sum_{n=0}^{\infty} y_{n}(x)=a+(b+F(a)) x-\int_{0}^{x}\left(\sum_{n=0}^{\infty} A_{n}\right) d s-\int_{0}^{x} \int_{0}^{t}\left(\sum_{n=0}^{\infty} B_{n}\right) d s d t
$$

Assuming absolute convergence of sums in (3.8), after rearranging Equation (3.8) we have

$$
\begin{aligned}
y_{0}(x)+y_{1}(x)+y_{2}(x)+\cdots=a & +(b+F(a)) x \\
& -\left(\int_{0}^{x} A_{0}(s) d s+\int_{0}^{x} \int_{0}^{t} B_{0}(s) d s d t\right) \\
& -\left(\int_{0}^{x} A_{1}(s) d s+\int_{0}^{x} \int_{0}^{t} B_{1}(s) d s d t\right)-\cdots .
\end{aligned}
$$

Now we take

$$
\begin{aligned}
& y_{0}(x)=a+(b+F(a)) x \\
& y_{1}(x)=-\int_{0}^{x} A_{0}(s) d s-\int_{0}^{x} \int_{0}^{t} B_{0}(s) d s d t, \\
& y_{2}(x)=-\int_{0}^{x} A_{1}(s) d s-\int_{0}^{x} \int_{0}^{t} B_{1}(s) d s d t
\end{aligned}
$$

and so on. Thus, we have the iteration

$$
\left\{\begin{array}{l}
y_{0}(x)=a+(b+F(a)) x \\
y_{n}(x)=-\int_{0}^{x} A_{n-1}(s) d s-\int_{0}^{x} \int_{0}^{t} B_{n-1}(s) d s d t, \quad n \in \mathcal{N} .
\end{array}\right.
$$

Now using the formula

$$
\int_{0}^{x} \int_{0}^{t} B_{n-1}(s) d s d t=\int_{0}^{x}(x-s) B_{n-1}(s) d s=x \int_{0}^{x} B_{n-1}(s) d s-\int_{0}^{x} s B_{n-1}(s) d s,
$$


the iteration (3.9) becomes

$$
\left\{\begin{array}{l}
y_{0}(x)=a+(b+F(a)) x \\
y_{n}(x)=-\int_{0}^{x} A_{n-1}(s) d s-x \int_{0}^{x} B_{n-1}(s) d s+\int_{0}^{x} s B_{n-1}(s) d s, \quad n \in \mathcal{N}
\end{array}\right.
$$

and finally $y(x)=\sum_{n=0}^{\infty} y_{n}(x)$.

The theoretical treatment of convergence of the decomposition method, together with the speed of convergence of this method, has been widely considered in the literature $[1,2,4,9,10,17]$.

\section{A numerical experiment}

In this section, we give a physically significant example in order to show the importance of our method. The van der Pol equation describes self-sustaining oscillations in which energy is fed into small oscillations and removed from large oscillations. This equation, which is familiar to everyone who has basic knowledge of electronics or mechanics, arises for example in the study of circuits containing vacuum tubes and is given by

$$
y^{\prime \prime}-\mu\left(1-y^{2}\right) y^{\prime}+y=0
$$

in which $\mu$ is the damping parameter. For the present example, we consider the initial conditions to be $y(0)=0, y^{\prime}(0)=1$. Therefore, we have $a=0, b=1, g(y)=y$ and $f(y)=-\mu\left(1-y^{2}\right)$, and hence $F(y)=-\mu\left(y-y^{3} / 3\right)$. The qualifying behaviour of the van der Pol equation has been investigated in the literature [12]. The reader can verify how our method circumvents the nonlinearity $\left(1-y^{2}\right) y^{\prime}$ and solves the equation in a very computationally efficient way. Putting $y=\sum_{n=0}^{\infty} y_{n}(x)$, the Adomian polynomials for $F(y)=-\mu\left(y-y^{3} / 3\right)$ are

$$
A_{0}=-\mu y_{0}+\frac{1}{3} \mu y_{0}^{3}, \quad A_{1}=-\mu y_{1}+\mu y_{0}^{2} y_{1}, \ldots
$$

and the Adomian polynomials for $g(y)=y$ are

$$
B_{0}=y_{0}, \quad B_{1}=y_{1}, \ldots
$$

Using the iteration (3.10),

$$
\begin{aligned}
y_{0}(x) & =x \\
y_{1}(x) & =-\int_{0}^{x}\left(-\mu s+\frac{1}{3} \mu s^{3}\right) d s-x \int_{0}^{x} s d s+\int_{0}^{x} s^{2} d s \\
& =\frac{1}{2} \mu x^{2}-\frac{1}{12} \mu x^{4}-\frac{1}{6} x^{3}, \\
y_{2}(x) & =\frac{1}{8} \mu^{2} x^{7}+\frac{11}{360} \mu x^{6}-\frac{7}{60} \mu^{2} x^{5}-\frac{1}{12} \mu x^{4}+\frac{1}{6} \mu^{2} x^{3}+\frac{1}{120} x^{5} .
\end{aligned}
$$


TABLE 1. The solution of (4.2) for $\mu=0.2$ and 0.5 approximated by the ADM and by Maple 12 .

\begin{tabular}{|c|c|c|c|c|c|c|}
\hline & \multicolumn{3}{|c|}{$\mu=0.2$} & \multicolumn{3}{|c|}{$\mu=0.5$} \\
\hline$x$ & ADM & Exact & Error & ADM & Exact & Error \\
\hline 0.1 & 0.1008367 & 0.1008367 & $0.00 \mathrm{E}+0$ & 0.1023669 & 0.1023672 & $3.00 \mathrm{E}-7$ \\
\hline 0.2 & 0.2026685 & 0.2026685 & $0.00 \mathrm{E}+0$ & 0.2088669 & 0.2088671 & $2.00 \mathrm{E}-7$ \\
\hline 0.3 & 0.3044240 & 0.3044241 & $1.00 \mathrm{E}-7$ & 0.3184341 & 0.3184345 & $4.00 \mathrm{E}-7$ \\
\hline 0.4 & 0.4049707 & 0.4049707 & $0.00 \mathrm{E}+0$ & 0.4297705 & 0.4297711 & $6.00 \mathrm{E}-7$ \\
\hline 0.5 & 0.5031302 & 0.5031302 & $0.00 \mathrm{E}+0$ & 0.5413486 & 0.5413469 & $1.70 \mathrm{E}-6$ \\
\hline 0.6 & 0.5977010 & 0.5977010 & $0.00 \mathrm{E}+0$ & 0.6514333 & 0.6514243 & $9.00 \mathrm{E}-6$ \\
\hline 0.7 & 0.6874851 & 0.6874849 & $2.00 \mathrm{E}-7$ & 0.7581329 & 0.7581097 & $2.32 \mathrm{E}-5$ \\
\hline 0.8 & 0.7713173 & 0.7713170 & $3.00 \mathrm{E}-7$ & 0.8594760 & 0.8594346 & $4.14 \mathrm{E}-5$ \\
\hline 0.9 & 0.8480960 & 0.8480958 & $2.00 \mathrm{E}-7$ & 0.9535096 & 0.9534573 & $5.23 \mathrm{E}-5$ \\
\hline 1.0 & 0.9168111 & 0.9168129 & $1.80 \mathrm{E}-6$ & 1.0384170 & 1.0383740 & $4.30 \mathrm{E}-5$ \\
\hline
\end{tabular}

TABLE 2. The solution of (4.2) for $\mu=1$ and 2 approximated by the ADM and by Maple 12 .

\begin{tabular}{|c|c|c|c|c|c|c|}
\hline \multirow[b]{2}{*}{$x$} & \multicolumn{3}{|c|}{$\mu=1$} & \multicolumn{3}{|c|}{$\mu=2$} \\
\hline & $\mathrm{ADM}$ & Exact & Error & ADM & Exact & Error \\
\hline 0.1 & 0.1049861 & 0.1049862 & $1.00 \mathrm{E}-7$ & 0.1104938 & 0.1104951 & $1.30 \mathrm{E}-6$ \\
\hline 0.2 & 0.2197546 & 0.2197565 & $1.90 \mathrm{E}-6$ & 0.2437836 & 0.2438159 & $3.23 \mathrm{E}-5$ \\
\hline 0.3 & 0.3436372 & 0.3436456 & $8.40 \mathrm{E}-6$ & 0.4017360 & 0.4018741 & $1.38 \mathrm{E}-4$ \\
\hline 0.4 & 0.4753143 & 0.4753214 & $7.10 \mathrm{E}-6$ & 0.5841367 & 0.5841299 & $6.80 \mathrm{E}-6$ \\
\hline 0.5 & 0.6126696 & 0.6126074 & $6.22 \mathrm{E}-5$ & 0.7874574 & 0.7854328 & $2.02 \mathrm{E}-3$ \\
\hline 0.6 & 0.7527138 & 0.7523829 & $3.30 \mathrm{E}-4$ & 1.0038030 & 0.9942659 & $9.50 \mathrm{E}-3$ \\
\hline 0.7 & 0.8916129 & 0.8906468 & $9.66 \mathrm{E}-4$ & 1.2203260 & 1.1935410 & $2.67 E-2$ \\
\hline 0.8 & 1.0248530 & 1.0228080 & $2.04 \mathrm{E}-3$ & 1.4193990 & 1.3654280 & $5.39 \mathrm{E}-2$ \\
\hline 0.9 & 1.1475590 & 1.1442000 & $3.30 \mathrm{E}-3$ & 1.5797800 & 1.4979750 & $8.18 \mathrm{E}-2$ \\
\hline 1.0 & 1.2549600 & 1.2507020 & $4.25 \mathrm{E}-3$ & 1.6789680 & 1.5885190 & $9.04 \mathrm{E}-2$ \\
\hline
\end{tabular}

Finally,

$$
\begin{aligned}
y(x)= & y_{0}(x)+y_{1}(x)+\cdots \\
= & x+\frac{1}{2} \mu x^{2}-\frac{1}{12} \mu x^{4}-\frac{1}{6} x^{3}+\frac{1}{8} \mu^{2} x^{7}+\frac{11}{360} \mu x^{6} \\
& -\frac{7}{60} \mu^{2} x^{5}-\frac{1}{12} \mu x^{4}+\frac{1}{6} \mu^{2} x^{3}+\frac{1}{120} x^{5}+\cdots .
\end{aligned}
$$

If we put $\mu=0$ in (4.2), then $y(x)$ is the Taylor series of $\sin (x)$, which is the exact solution of $y^{\prime \prime}+y=0$. We have compared the numerical solutions obtained by 
the ADM, for the number of iterations $n=3$, with the exact solutions obtained by Maple 12 for different values of $\mu$ in Tables 1 and 2. Results are not shown for $x=0$ as (4.2) shows that $y(0)=0$ for all $\mu$.

One can easily see that the larger the value of $\mu$, the bigger the error in the numerical solution. That is because as $\mu$ increase the equation becomes more and more nonlinear.

\section{Acknowledgements}

The authors would like to express their gratitude to the Editor-in-Chief and the anonymous referee for their valuable comments and recommendations which improved the quality of this work significantly.

\section{References}

[1] K. Abbaoui and Y. Cherruault, "Convergence of Adomian's method applied to differential equations”, Comput. Math. Appl. 28 (1994) 103-109.

[2] K. Abbaoui and Y. Cherruault, "New ideas for proving convergence of decomposition methods", Comput. Math. Appl. 29 (1995) 103-108.

[3] K. Abbaoui, Y. Cherruault and V. Seng, "Practical formula for the calculus of multivariable Adomian polynomials", Math. Comput. Modelling 22 (1995) 89-93.

[4] K. Abbaoui, M. J. Pujol, Y. Cherruault, N. Himoun and P. Grimalt, "A new formulation of Adomian method: convergence result", Kybernetes 30 (2001) 1183-1191.

[5] G. Adomian, "A review of the decomposition method in applied mathematics", J. Math. Anal. Appl. 135 (1988) 501-544.

[6] G. Adomian, Solving Frontier problems of Physics: the decomposition method (Kluwer Academic Publishers, Boston, 1994).

[7] E. Babolian and J. Biazar, "On the order of convergence of Adomian method", Appl. Math. Comput. 130 (2002) 383-387.

[8] E. Babolian and Sh. Javadi, "New method for calculating Adomian polynomials", Appl. Math. Comput. 153 (2004) 253-259.

[9] Y. Cherruault, “Convergence of Adomian's method”, Kybernetes 8 (1988) 31-38.

[10] Y. Cherruault and G. Adomian, "Decomposition methods: a new proof of convergence", Math. Comput. Modelling 18 (1993) 103-106.

[11] C. Chun, "A new iterative method for solving nonlinear equations", Appl. Math. Comput. 178 (2006) 415-422.

[12] J. M. Dixon, J. A. Tuszyfiski and D. Sept, "Orthogonal trajectories and analytical solutions of the van der Pol equation without forcing”, Phys. Lett. A 239 (1998) 65-71.

[13] J. Guckenheimer, "Dynamics of the van der Pol equation", IEEE Trans. Circuits Syst. 27 (1980) 983-989.

[14] J. K. Hale, Ordinary differential equations (Wiley, New York, 1980).

[15] M. M. Hosseini and H. Nasabzadeh, "On the convergence of Adomian decomposition method", Appl. Math. Comput. 182 (2006) 536-543.

[16] D. Kaya and S. M. El-Sayed, "A numerical implementation of the decomposition method for the Liénard equation”, Appl. Math. Comput. 171 (2005) 1095-1103.

[17] A. Repaci, "Nonlinear dynamical systems: on the accuracy of Adomian's decomposition method", Appl. Math. Lett. 3 (1990) 35-39.

[18] A. M. Wazwaz, "A reliable modification of Adomian decomposition method", Appl. Math. Comput. 102 (1999) 77-86.

[19] A. M. Wazwaz, "A new algorithm for calculating Adomian polynomials for nonlinear operators", Appl. Math. Comput. 111 (2000) 53-69.

[20] Y. Zhu, Q. Chang and S. Wu, "A new algorithm for calculating Adomian polynomials", Appl. Math. Comput. 169 (2005) 402-416.

[21] D. Zwillinger, Handbook of differential equations (Academic Press, San Diego, CA, 1992). 